\title{
Modelling a Sustainable Cooperative Healthcare: An Interoperability-driven Approach
}

\author{
Ovidiu Noran ${ }^{1}$, Hervé Panetto ${ }^{2,3}$ \\ ${ }^{1}$ School of ICT, Griffith University, Australia, ${ }^{2}$ CNRS, CRAN UMR 7039, France, \\ ${ }^{3}$ University of Lorraine, CRAN UMR 7039, France \\ 10.noran@griffith.edu.au, ,33herve.panetto@univ-lorraine.fr
}

\begin{abstract}
Modern healthcare is confronted with serious issues that are threatening its viability and sustainability. Increasing costs and complexity, global population ageing and pandemics are major culprits of the healthcare quandary. In this context, effective interoperability of the participants in the healthcare effort becomes paramount. However, this is also a major challenge as unfortunately, healthcare institutions typically feature strong hierarchy and heterogeneity. As the pressure on healthcare resources and management cost is constantly increasing, governments can no longer rely on outdated 'silo' paradigms for managing population wellbeing. New cooperative and integrated models and procedures taking into account all essential cooperation aspects, elements, participants and their life cycle are necessary to drive cooperative healthcare sustainability. Based on previous research and applications, this paper argues that the necessary artefacts can be built using a life cycle-based, holistic paradigm enabled by advances in Interoperability, Enterprise Architecture and Collaborative Networks research and practice. The proposed modelling approach aims to provide a solid base for sustainable solutions to long and short-term challenges to population health and well-being.
\end{abstract}

Keywords: Healthcare, Sustainability, Interoperability, Enterprise Architecture, Collaborative Networks

\section{Introduction}

Worldwide, healthcare is under escalating pressure from population ageing, drugresistant pandemics, increasing complexity and rising costs. In this context, silo-type legacy governance models have lost relevance as interoperability and cooperation are sine qua non requirements for survival and progress in a global environment.

Unfortunately, there are significant challenges in managing the internal and external collaboration of the typically heterogeneous set of participants involved in the healthcare endeavour. New integrated models, methods and tools are required in order to enable proper inter-professional and inter-organisational cooperation, so as to meet these serious long and short term healthcare challenges.

In addition, sustainability (seen in manufacturing as the creation of products using processes that minimize environmental impact, conserve energy and natural 
resources, are safe for employees, communities and consumers while also being economically sound [1]) can be extrapolated to the healthcare domain in order to assess its capacity to endure in an ever-changing, increasingly complex environment.

Previous research has investigated specific interoperability aspects [2, 3] and the role of Collaborative Networks (CN) [4] and Enterprise Architecture (EA) [5] concepts and methodologies in supporting generic collaboration efforts between heterogeneous organisations [6, 7]. This paper aims to build on the previous results by focusing on the healthcare area in a multifaceted and integrated manner.

\section{Challenges in Healthcare Management Collaboration}

Healthcare has made significant advances in the last century, such as the development of vaccines, eradication of serious diseases and large reductions in communicable disease epidemics and chronic diseases [8,9]. While solving some very important problems, some of these advances have unfortunately also contributed to a new set of challenges faced by the public and private healthcare infrastructure and organisations. For example, population growth and ageing triggered by increased longevity [9] reflects mankind progress and provides benefits [10] but also brings significant social security and healthcare challenges [11]. Another major concern are the increasingly complex health incidents (e.g. pandemics) owing to new strains of diseases [12], climate change [13] and population displacements fuelled by regional conflicts.

Whereas healthcare as a system has become somewhat more organised, it has also become more expensive, complex and difficult to manage. The intricate nature of the organisations involved presents significant impediments to technology transfer and diffusion [14] that includes interactional user resistance to the new systems [15]. Research in the field confirms however that the main barriers to healthcare cooperation are of semantic, pragmatic and organisational nature [16-19]. Thus, collaboration between healthcare effort participants does not automatically occur. It must be “constructed, learned, and once established, protected” [16].

The divergent perceptions and expectations of the parties involved [18], owing to a traditionally strong hierarchy and marked difference in status between partners [19], can be best dealt with by the higher ranking participants. They can promote collaboration and trust by employing a participatory and inclusive approach [20] which will also build a beneficial sense of security [21].

Inter-professional and inter-organisational collaborative healthcare is encouraged in various medical and emergency response reports, conferences and journals (e.g. [22-26]) as well as in international projects. For example, the BRAID project [27] advocates the necessity for collaborative healthcare ecosystems [28] supported by integrated assistive services and infrastructure [26]. Unfortunately however, the extent of actual cooperation in healthcare is still limited as efficient long-term healthcare collaboration requires that organisational cultures, processes and resources of the participants acquire suitable preparedness [22, 29, 30], with ethics playing a prominent role [31, 32]. This requires access to a plethora of multidisciplinary information and knowledge; as such, participatory analysis and design [33] represent important collaborative healthcare enablers that help integrate all necessary scientific, administrative, social and political aspects into a whole-system approach [23, 29, 34]. 
In a typical health incident scenario, often there is a tendency of the higher ranking and more powerful organisation(s) to override or exclude some participants, adopting a 'central command' approach rather than a cooperative one [35]. This is not desirable as successful disaster management relies on a wide range of community, economic, social-psychological, and political resources.

\section{Interoperability for Sustainable Cooperative Healthcare}

The concept of interoperability is often used as a measure of cooperation capability assigned to systems [36] and to contrast mere information exchange between those systems, including in the healthcare domain [37, 38]; inter- and intra-organisational interoperability enable companies healthcare effort participants to cope with and sustain in the modern networked, dynamic and challenging environment [39]. Healthcare systems interoperability analysis must include some important aspects, such as extent, approach and aspects covered. For example, as shown in previous research [2, 7], an interoperability degree close to total integration would imply a loss of autonomy, which is undesirable (e.g. in crisis situations where response teams may get isolated). On the other extreme, minimal interoperability (compatibility) of the healthcare or health crisis management effort participants is unsuitable and can only serve as a good starting point. The desirable degree of interoperability lies between these depending on the specific healthcare or health crisis management endeavour (Fig. 1 right). Importantly, agile organisations are able to maintain a high degree of interoperability while adapting to changes in the environment (see Fig. 1 left).

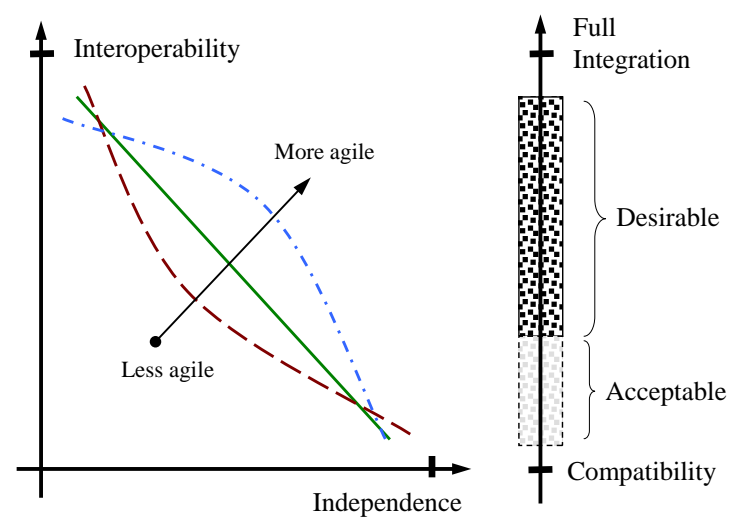

Fig. 1 Interoperability issues and approach in disaster management (based on [2, 7])

The 'full integration' and 'federalisation' interoperability options specified in ISO14258 [40] do not appear to achieve the desired results in healthcare due to pronounced organisational heterogeneity and the impracticality to negotiate in the limited time available during a disaster event. The unified approach [ibid.] appears to be more suitable to this domain as it assumes that ontology is negotiated in advance so as to achieve semantic interoperability; notwithstanding advances in negotiations and ontology research [41, 42], in our opinion the most efficient method to achieve 
unification is for the organisations to 'spend time together' in order to agree on the meanings associated with the concepts used to exchange knowledge.

Interoperability aspects are provided by various standards [40] and frameworks (e.g. European Interoperability Framework (EIF)[43], IDEAS project [44], ATHENA Interoperability Framework (AIF)[45] and the INTEROP Network of Excellence (NoE) Interoperability Framework [46]). All these frameworks have overlapping and complementary areas; in addition, it is important that combinations of aspects are also considered. Therefore, a combined model has been constructed and applied for identifying the most relevant aspects for healthcare interoperability (see Fig. 2).

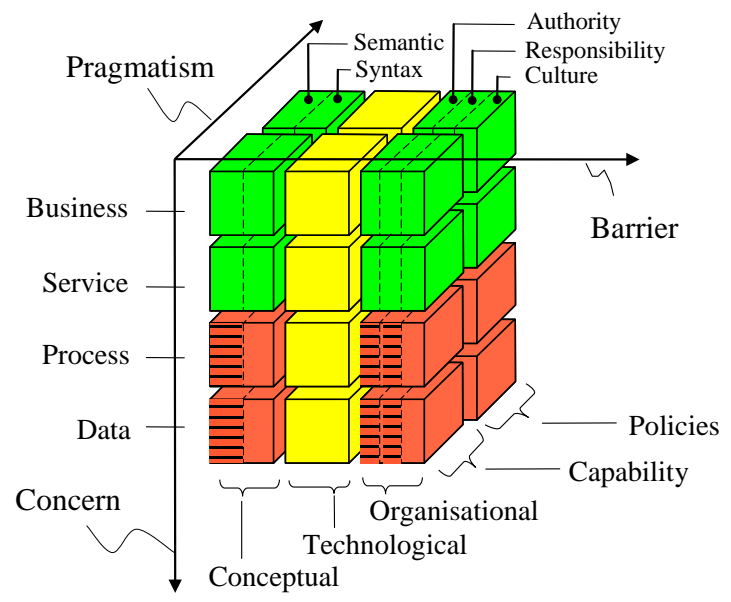

Legend: $\equiv=$ critical $\square$ = primary $\square=$ secondary $\square$ = tertiary

Fig. 2. An interpretation of the INTEROP NoE Interoperability Framework [46] enriched with concepts from ISO14258, EIF, IDEAS, ATHENA AIF, [2] and [7].

The pragmatic interoperability aspect $[3,47]$ relates to the willingness of the participants to interoperate; the two main components, capability and policies (see Fig. 2) suggest that the healthcare organisations need to gain appropriate cooperation preparedness that must be endorsed and supported by the executive.

The semantic aspect of the data and process areas has a typically high priority: in a disaster event, the capability to extract and interpret data from heterogeneous sources is essential for situational awareness preventing life-threatening situations. Therefore, prior agreements on data format and especially on data meaning are essential. This is also applicable to long term collaborative healthcare problems as illustrated by current Electronic Health Records (EHR) semantic interoperability problems [48, 49].

The organisational interoperability aspect is an essential aspect in healthcare, where participants exhibit significant structure diversity; thus, the responsibility and authority interoperability aspects [43, 46] are another critical area identified in Fig. 2. The roles and hierarchy in long and short term healthcare cooperative structures must be commonly understood and accepted if appropriate synergy and focus is to be achieved. The third organisational interoperability component, i.e. cultural [3], is notoriously hard to tackle. Regular immersion of the participant organisations in each other's cultures ('co-habitation') may assist in this direction. 


\section{Collaborative Networks for Sustainable Semantic, Pragmatic and Organisational Interoperability}

The concept of networks in disaster management and recovery has been advocated, studied and applied to some extent for a number of years with mixed results (e.g. [35, 50, 51]). Unfortunately, these attempts appear to have two main shortcomings. Firstly, they use untested models focusing on specific aspects, rather than employing a proven set of integrated models in a whole-system approach. Secondly, they seem to pay less attention to the life cycle aspect of the participant organisations, networks and other relevant entities, including the disaster event/s.

In researching the healthcare-specific interoperability issues, it has been observed that the challenges identified describe a situation similar to that of commercial enterprises who, owing to a global business environment, find themselves compelled to tackle projects requiring resources beyond their own. The usual solution to this problem is to set up (or join) so-called 'Collaborative Networks' (CNs) that act as breeding environments for Virtual Organisations (VOs) who are promptly created in order to bid for and complete projects requiring combined resources and know-how. The view of CNs as commitment-based social systems that absorb uncertainty and reduce complexity [52] supports their use in the typically elaborate long and short term healthcare projects.

The CNs and VOs set up for the healthcare domain would have specific features. Thus, the competitive motivations of commercial $\mathrm{CN}$ participants guiding their decisions to create / join / remain / leave the network would transform into the need to cope with increasingly complex health challenges and healthcare systems. Here, a 'Health Management' CN (HMCN) would create 'Health Management' VOs (HMVOs) for long term projects (e.g. as described in [53]), or task forces (HMTFs) for shorter term and more intense events (e.g. pandemics). The use of reference models, customary in commercial CNs, may be limited here due to diversity [54].

For a HMCN to function, scientific, faith and community representatives and all relevant non-governmental and volunteer organisations must also be included in the setup and operation of the HMCN, in addition to the typical participants such as hospitals, allied healthcare [55], fire and rescue services, etc.

Adopting a CN approach for health disaster management provides benefits going beyond mere technical and syntactic-type interoperability. Thus, the participants in a HMCN have the time and suitable environment to overcome the previously described semantic, pragmatic and organisational interoperability barriers and achieve the required preparedness. This is essential in the prompt and successful setup of HMTFs for disasters and in the creation and operation of continuing HMVOs for long term healthcare challenges such as population ageing.

\section{The Enterprise Architecture Role in a Holistic and Integrated Approach towards Sustainable Interoperability}

Healthcare collaboration requirements are multi-faceted and inherently linked to the life cycle phase(s) of the organisations; it is therefore essential that the proposed 
networked collaboration analysis is performed in an aspect-integrated manner and in a life cycle context so that the interoperability is achieved, but in a sustainable manner. It is hereby argued that an optimal way to integrate the life cycle aspect in a sustainable collaborative healthcare scenario is by using an EA perspective.

EA is seen in this context as a holistic change management paradigm that bridges management and engineering best-practice, providing the "[...] key requirements, principles and models that describe the enterprise's future state. [...] EA comprises people, processes, information and technology of the enterprise, and their relationships to one another and to the external environment" [5]. This EA definition reinforces the view of CNs as social systems composed of commitments [52] and healthcare as socio-technical systems with voluntaristic people [56] in a complex organisational, political and behavioural context $[15,57,58]$. As such, EA is capable of providing a framework integrating all necessary aspects in a life cycle-based set of models ensuring the consistency and sustainability of complex projects.

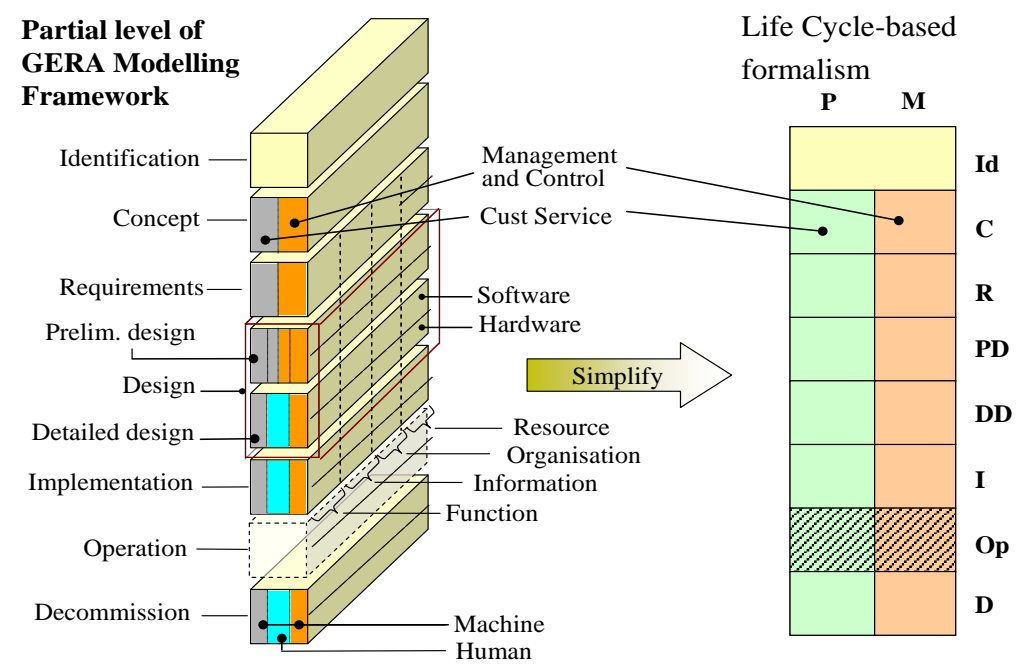

Fig. 3 Using Enterprise Architecture Modelling Framework elements

In this example, we have selected the modelling framework (MF) provided by 'GERAM' (Generalised Enterprise Reference Architecture and Methodology), described in ISO 15704:2005 [59]. This MF provides a large set of aspects, importantly including life cycle, management, organisation and human. Aspect-based subsets of the GERA MF can be turned into life cycle-based constructs used to produce business models requiring a life cycle-based analysis. For example, aspects previously identified as significant in improving cooperation in disaster management (e.g. function, information, resources, organisation) but also additional supporting viewpoints like management vs. operations, automation boundary / human extent, etc) can be represented as shown in Fig. 3, left. Aspects can also be separated to promote clarity; for example, the 2-dimensional structure shown in Fig. 3 right is used to focus on the product/service and management viewpoints in a life cycle context. 

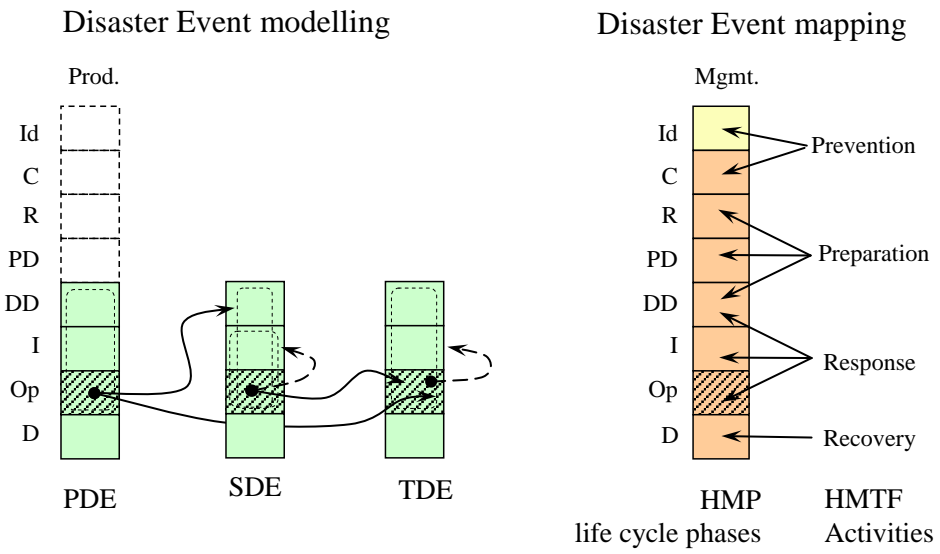

Legend:

PDE / SDE / TDE = Primary / Secondary / Tertiary Disaster Event;

HMP = Health Mgmt Project; HMTF / HMTF = Health Management Task Force

$\square=$ Production view $\square=$ Management view $\bullet-\rightarrow=$ Possible scenario

Fig. 4 Disaster event mapping and modelling using a life cycle-based modelling construct

Figure 4 right shows the use of GERA's MF life cycle viewpoint to define and map the life cycle phases of a health management project (HMP) dealing with a health incident on typical health disaster management activities [60]. For the sake of clarity, Fig. 4 does not consider the time dimension (life history); this can be represented orthogonally to illustrate the actual life cycle sequence (i.e, the life cycle stages).

The left hand side of Fig. 4 shows the use of the above-described formalism to represent a disaster event, focusing only on the relevant life cycle phases and relations to other events' life cycles. Thus, Fig. 4 left shows how a Primary Disaster Event (PDE) can trigger or influence secondary/tertiary etc events (SDE, TDE). For example, an earthquake event (PDE) can trigger a tsunami (SDE) that can in turn trigger a partial nuclear meltdown or a pandemic (TDE). This modelling approach can also show PDEs influencing TDEs directly and 'chain reaction'-type events (arrows from Operation to Implementation within same entity).

\section{Life Cycle Integrated Modelling of Collaborative Healthcare Interoperability Requirements}

Successful integration modelling of collaborative healthcare depends on an inclusive approach involving all the network participants [34]. The proposed modelling method supports this audience variety with graphical models and complexity management. For example, Fig. 5 uses the modelling construct shown in Fig. 3 right to depict data interoperability requirements of HMCN and HMTF / HMVO creation and operation. The arrows in Fig. 5 show data-specific influences and contributions requiring interoperability among the entities involved in the long and short term healthcare 
endeavour. Thus, healthcare organisations HO (e.g. hospitals), allied health professionals (AHP) and scientific, faith, etc and other communities representatives (CSFR) all contribute to the design and operation of a HMCN in its various life cycle phases and thus require proper data interoperability, as detailed in the figure.

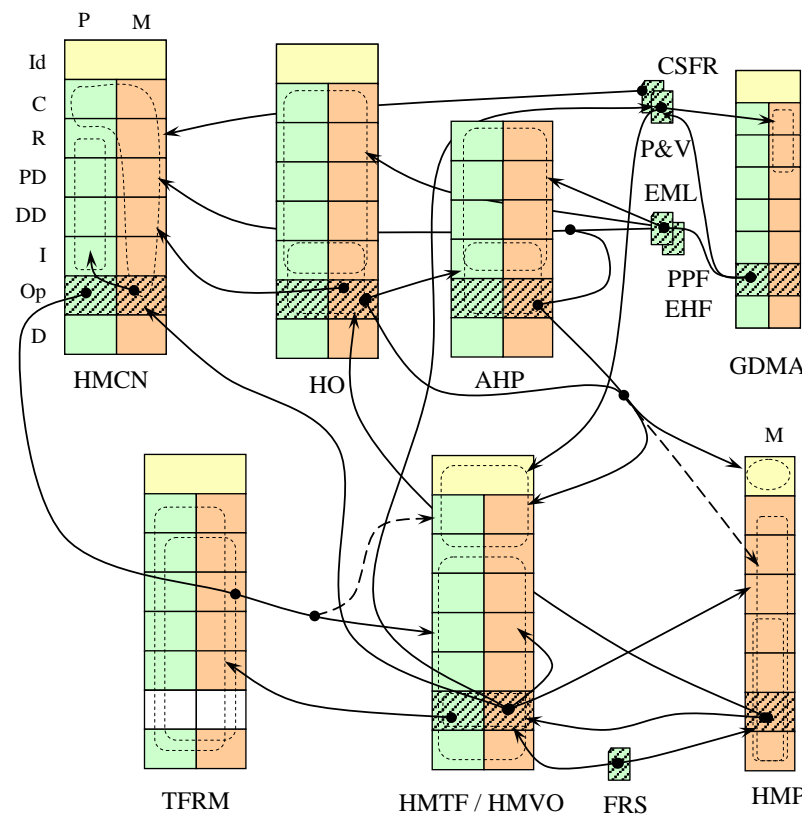

Life cycle phases: $I d=I d e n t i f i c a t i o n ; ~ C=$ concept; $R=$ requirements,

$\mathrm{PD}=$ preliminary design, $\mathrm{DD}=$ detailed design, $\mathrm{I}=$ implementation, $\mathrm{Op}=$ operation, $\mathrm{D}=$ decommissioning. Other aspects: $\mathrm{P}=$ Production / Service, $\mathrm{M}=$ management.

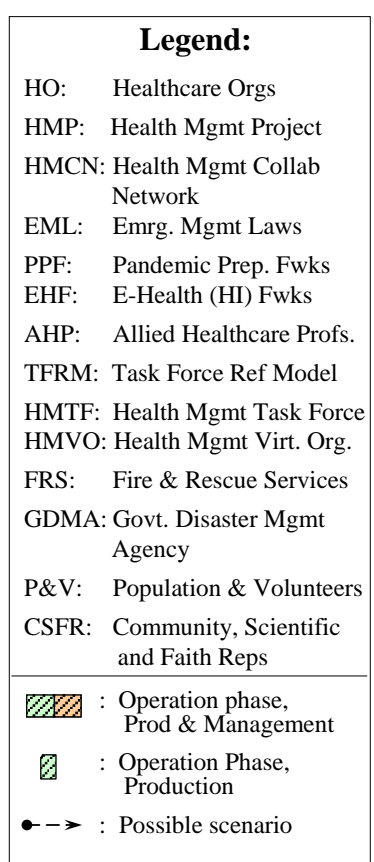

Fig.5 Data interoperability requirements in a possible HMCN and HMVO / HMTF scenario

These requirements may also extend directly to the design and operation of the HMTFs / HMVOs created by the HMCN, and to the health management projects (HMPs) created by the HTMF/ HMVOs to deal with long and short term healthcare incidents and challenges. Influences and contributions requiring interoperability also come from 'non-physical' artefacts such as emergency management laws (EML), pandemic preparedness (PPF), or e-health strategies / frameworks (EHF) [61, 62]. Properly aggregated and understandable information must be provided by HTMFs / HMVOs to population and volunteers $(\mathrm{P} \& \mathrm{~V})$. This is paramount as in an emergency it will directly decide the amount of lost property and casualties; past experience has shown that $\mathrm{P} \& \mathrm{~V}$ must receive but also understand, believes and act on HMTF warnings and directives. The arrow from HMTF / HMVO's Management side of the Operation life cycle phase to some of its upper phases represents a 'limited agility' requirement allowing the HMTF to remain interoperable in the face of changing conditions on the ground typical to disaster-type events. However, any major HMTF / HMVO interoperability reconfiguration (e.g. involving Requirements or Architectural Design life cycles) must involve the HMCN participants' and other entities' input. 
Note that high-level models such as depicted in Fig.5 do not aim to provide all the details necessary for actual implementation. Their purpose is rather to provide a 'checklist' of the interoperability requirements of the specific collaborative healthcare endeavour and highlight relevant interactions, all in a life cycle context. Such models can represent various autonomy and agility scenarios for the collaborative healthcare participants. Once stakeholder consensus on present and future states has been achieved, these models can be evolved into design and implementation blueprints.

\section{Conclusions}

Politics, hierarchy, diverging perceptions, lack of trust, dissimilar organisational structures and cultures and limited life cycle perspective of the healthcare participants' roles and interactions all inhibit collaboration. This paper has proposed a multidisciplinary solution that draws upon a rich repository of Interoperability, Enterprise Architecture and Collaborative Networks research and practice results.

The paper makes a theoretical contribution by highlighting the synergy between several research areas to advance collaborative healthcare and a practical contribution by providing an example of how interoperability aspects can be analysed and improved using $\mathrm{CN}$ concepts from an EA perspective in order to model a collaborative healthcare solution to the current health and well-being challenges.

\section{References}

1. International Trade Administration. How does Commerce define Sustainable Manufacturing? 2010; http://www.trade.gov/competitiveness/how_doc_defines_SM.asp.

2. Panetto, H., Towards a classification framework for interoperability of enterprise applications. Int. J. of Comp. Integrated Manufacturing, 2007. 20: p. 727-740.

3. Whitman, L. and H. Panetto, The Missing Link: Culture and Language Barriers to Interoperability. Annual Reviews in Control, 2006. 30(2): p. 233-241.

4. Camarinha-Matos, L. and H. Afsarmanesh, Collaborative Networks: A new scientific discipline. Journal of Intelligent Manufacturing, 2005. 16: p. 439-452.

5. Gartner Research. IT Glossary. 2012 [2012]; http://www.gartner.com/technology/itglossary/enterprise-architecture.jsp.

6. Noran, O., Towards A Collaborative Network Paradigm for Emergency Services. Adaptation and Value Creating Collab. Networks - IFIP Adv in ICT, 2011. 362: p. 477-485.

7. Noran, O. and P. Bernus, Effective Disaster Management: An Interoperability Perspective. Lecture Notes in Computer Science, 2011. 7046: p. 112-121.

8. Fielding, J.E., Public Health in the Twentieth Century: Advances and Challenges. Annual Reviews in Public Health, 1999. 20: p. xiii-xxx.

9. World Health Organization. The world health report 1998---life in the 21st century: a vision for all. 1998 [cited 2013 April]; http://www.who.int/whr/1998/en/whr98_en.pdf.

10.Healy, J. The Benefits of an Ageing Population - Discussion Paper 63. The Australia Institute 2004 [cited 2013 April]; http://www.tai.org.au/documents/dp_fulltext/DP63.pdf.

11.International Labour Organisation. Ageing societies: The benefits, and the costs, of living longer. 2009 [2013]; http://www.ilo.org/global/WCM_041965/lang--en/index.htm.

12.Kilbourne, E.D., Influenza Pandemics of the 20th Century. Emerg. Inf. Dis., 2006. 12(1). 
13.Donohoe, M., Causes and health consequences of environmental degradation and social injustice. Social Science and Medicine, 2003. 56(3): p. 573-587.

14.Southon, F.C., C. Sauer, and C.N. Grant, Information technology in complex health services: organizational impediments to successful technology transfer and diffusion. J Am Med Inform Assoc., 1997. 4(2): p. 112-124.

15.Markus, M.L., Power, politics \& MIS implementation. Comm ACM, 1983. 26: p. 430-444.

16.Wilson, K., et al., Nurse Practitioners' Experience of Working Collaboratively with General Practitioners and Allied Health Professionals in NSW, Australia. Australian Journal of Advanced Nursing, 2005. 23(2): p. 22-27.

17.Braude, R.M., People and Organizational Issues in Health Informatics. J Am Med Inform Assoc., 1997. 4(2): p. 150-151.

18.Krogstad, U., D. Hofoss, and P. Hjortdal, Doctor and nurse perception of interprofessional co-operation in hospitals. Int J. for Quality Health Care, 2004. 16(6): p. 491-497.

19.Ramanujam, R. and D.M. Rousseau, The Challenges Are Organizational, Not Just Clinical. Journal of Organizational Behavior, 2006. 27(7): p. 811-827.

20.Baker, D., R. Day, and E. Salas, Teamwork as an essential component of high reliability organizations. Health Services Research, 2006. 41(4): p. 1577-1598.

21.Nembhard, I.M. and A.C. Edmondson, Making It Safe: The Effects of Leader Inclusiveness and Professional Status on Psychological Safety and Improvement Efforts in Health Care Teams. Journal of Organizational Behavior, 2006. 27(7): p. 941-966.

22.Kapucu, N., T. Arslan, and F. Demiroz, Collaborative emergency management and national emergency management network. Disaster Prev. and Management, 2010. 19(4): p. 452-468.

23.Utah Department of Health. Governor's Task Force for Pandemic Influenza Preparedness Final report to Governor. 2007 [cited 2013 April]; Available from: http://pandemicflu.utah.gov/docs/PandInfluTaskforceFinalReport.pdf.

24.Waugh, W.L. and G. Streib, Collaboration and Leadership for Effective Emergency Management. Public Administration Review, 2006. 66(s1): p. 131-140.

25.Hughes, R.G., Chapter 33: Professional Communication and Team Collaboration, in Patient Safety and Quality: An Evidence-Based Handbook for Nurses. 2008, Agency for Healthcare Research and Quality,: Rockville, MD.

26.Sansoni, J., et al. An Assessment Framework for Aged Care. 2012 April [cited 2013 April]; Available from: Centre for Health Service Development, University of Woolongong.

27.BRAID. Bridging Research in Ageing and ICT Development - Consolidated Vision of ICT and Ageing. 2011 [2013]; http://auseaccess.cis.utas.edu.au/sites/default/files/D4.2Final.pdf.

28.Holzman, T.G., Computer-human interface solutions for emergency medical care. Interactions, 1999. 6(3): p. 13-24.

29.World Health Organisation. Pandemic Influenza preparedness Framework. 2011 [2013]; Available from: http://whqlibdoc.who.int/publications/2011/9789241503082_eng.pdf.

30.U.S. Dept of Health and Human Services. HHS Pandemic Influenza Plan. 2005 [2013]; http://www.flu.gov/planning-preparedness/federal/hhspandemicinfluenzaplan.pdf.

31.Thompson, A.K., et al., Pandemic influenza preparedness: an ethical framework to guide decision-making. BMC Medical Ethics, 2006. 7(12).

32.NZ Ethics Advisory Committee. Ethical Values for Planning for and Responding to a Pandemic in New Zealand. 2006 [2013];http://neac.health.govt.nz/sites/neac.health.govt.nz/ pandemic-planning-and response.pdf.

33.Kristensen, M., M. Kyng, and L. Palen. Participatory Design in Emergency Medical Service: Designing for Future Practice. in CHI 2006. 2006. Montréal, Québec / Canada.

34.Moghadas, S.M., et al., Managing public health crises: the role of models in pandemic preparedness. Influenza Other Respi Viruses, 2008. 3(2): p. 75-79.

35.Waugh, W.L., Coordination or Control: Organizational Design and the Emergency Management Function. Int J. of Disaster Prevention and Management, 1993. 2(4): p. 17-31. 36.DoD Architecture Framework Working Group. DoD Arch Fwk Ver 1.0. 2004 [cited 2007]. 
37.Ray, P. and B. Vargas, Interoperability of hospital IS, in Proc. of the $5^{\text {th }}$ Int. Workshop on Enterprise Networking and Computing in Healthcare Industry. 2003: Santa Monica, CA.

38.Fridsma, D. Interoperability vs Health Information Exchange: Setting the Record Straight. 2013 [cited 2013 May]; Available from: http://www.healthit.gov/buzz-blog/meaningfuluse/interoperability-health-information-exchange-setting-record-straight/.

39.Molina, A., et al., Enterprise Integration and Networking: Challenges and Trends. Studies in Informatics and Control, 2007. 16(4).

40.ISO, ISO14258 Industrial Automation Sys Concepts and Rules for Enterprise Models. 2005.

41.Farquhar, A., et al., Collaborative Ontology Construction for Information Integration. Technical Report KSL-95-63. 1995, Stanford University: Knowledge Systems Laboratory.

42.Pinto, H., A. Prez, and J. Martins, Some Issues on Ontology Integration (Proceedings of IJCAI-99). 1999, Stockholm, Sweden.

43.EIF, European interoperability framework for pan-European eGovernment services. Luxembourg: Interoperable Delivery of European eGovernment Services to public Administrations, Businesses and Citizens (IDABC). 2004.

44.IDEAS,Project Deliverables (WP1-WP7) Public repts 2003 [2011] www.ideas-roadmap.net

45.ATHENA State of the art of Enterprise Modelling Techniques and Technologies to Support Enterprise Interoperability. Deliv D.A1.1.1 2004 [cited 2012]; http://www.athena-ip.org.

46.Chen, D., Practices, principles and patterns for interop. INTEROP-NOE, Interoperability Research for Networked Enterprises Network of Excellence, $n^{\circ}$ IST 508-011, 2005.

47.Tsagkani, C., Inter-Organizational Collaboration on the Process Layer, in Proc. of the IFIP/ACM SIGAPP INTEROP-ESA Conference. 2005: Geneva, Switzerland.

48.Beale, T. and S. Heard, An Ontology-based Model of Clinical Information. S. Stud Health Technol Inform, 2007. 129 (Pt.1): p. 760-764.

49.Rector, A., R. Qamar, and T. Marley, Binding Ontologies \& Coding systems to Electronic Health Records and Messages. Applied Ontology, 2009. 4(1).

50.Australian Psychological Society. Disaster Response Network (DRN). 2013 [cited 2013]; Available from: http://www.psychology.org.au/medicare/drn/.

51.Cooper, S., et al., Collaborative practices in unscheduled emergency care: role and impact of the emergency care practitioner - qualitative, quantitative and summative fndings. Emergency Medicine Journal, 2007(24): p. 625-633.

52.Neumann, D., L.A. de Santa-Eulalia, and E. Zahn. Towards a Theory of Collaborative Systems. in Proceedings of the $12^{\text {th }}$ IFIP - PROVE'11. 2011. São Paulo / Brazil: 362.

53.Noran, O., Collaborative networks in the tertiary education industry sector: a case study. International Journal of Computer Integrated Manufacturing, 2013. 1-2: p. 29-40.

54.Tierney, K. and E.L. Quarantelli, Needed Innovation in the Delivery of Emergency Medical Services in Disasters: Present and Future. Disaster Management, 1989. 2(2): p. 70-76.

55.Queensland Health. Allied health career structure. 2012 [cited 2013]; Available from: http://www.health.qld.gov.au/allied/career-structure.asp.

56.McGregor, D., The Human Side of Enterprise. 1960, New York: McGraw-Hill.

57.Keen, P.G.W. and M. Scott Morton, Decision Support Systems: An Organisational Perspective. 1978, Reading, Massachussetts: Addison-Wesley.

58.Iivari, J., A Paradigmatic Analysis of Contemporary Schools of IS Development. Eur. J. Information Systems, 1991. 1(4): p. 249-272.

59.ISO/IEC, Annex C: GERAM, in ISO/IS 15704:2000/Amd1:2005: Industrial automation systems - Requirements for enterprise-reference architectures and methodologies. 2005.

60.Australian Government. Attorney's General's Office - Emergency Management in Australia. 2011 [2011]; Available from: http://www.ema.gov.au/.

61.Council of Australian Governments. National E-health Strategy. 2008 [cited 2013 April]; http://www.ahmac.gov.au/cms_documents/national\%20e-health\%20strategy.pdf.

62.eHealth Task Force Report - European Commission. Redesigning health in Europe for 2020. 2012 [cited 2013 May]; http://www.president.ee/images/stories/pdf/ehtf-report2012.pdf. 